\title{
Alzheimer's Disease Prediction Model Using Demographics and Categorical Data
}

\author{
https://doi.org/10.3991/ijoe.v15i15.11472 \\ Aunsia Khan ( $\left.{ }^{\varpi}\right)$, Muhammad Usman \\ Shaheed Zulfikar Ali Bhutto Institute of Science and Technology, \\ Islamabad, Pakistan \\ aunsia@szabist-isb.edu.pk
}

\begin{abstract}
Diagnosing Alzheimer's disease (AD) is usually difficult, especially when the disease is in its early stage. However, treatment is most likely to be effective at this stage; improving the diagnosis process. Several AD prediction models have been proposed in the past; however, these models endure a number of limitations such as small dataset, class imbalance, feature selection methods etc which place strong barriers towards the accurate prediction. In this paper, an $\mathrm{AD}$ prediction model has been proposed and validated using categorical dataset from National Alzheimer's Coordination Center (NACC). The different categories such as Demographics, Clinical Diagnosis, MMSE \& Neuropsychological battery, is preprocessed for important features selection and class imbalance. A number of predominant classifiers namely, Naïve Bayes, J48, Decision Stump, LogitBoost, AdaBoost, and SDG-Text have been used to highlight the superiority of a classifier in predicting the potential AD patients. Experimental results revealed that Bayesian based classifiers improve AD detection accuracy up to $96.4 \%$ while using Clinical Diagnosis category.
\end{abstract}

Keywords-Alzheimer's disease prediction, Naïve Bayes, Class imbalance, Machine learning, early diagnosis.

\section{$1 \quad$ Introduction}

In health sector, Alzheimer's disease has become one of the major concerns for the neurologists. It is considered to be a sixth major cause of overall deaths, while a third major cause of death for elderly people in America [1]. AD is caused by the retention of amyloid plaques and neuro fibrillary tangles in the brain resulting in decreased mental ability and reasoning, effecting daily life tasks [2]. Till date, there is no medication that can reverse or stop the damages caused by the disease, for this reason considerable progress has been reported in only non-pharmacological treatments [3]. Therefore, the timely treatment and strategies for the early detection of AD for progression delay is of fundamental importance. Furthermore, machine learning techniques have widely been utilized for the analysis and identification of the disease patterns [4] [5]. For the analysis of AD prediction, ML techniques are recently applied [6] [7] [8]. The treatment plans and speed in progression relies heavily on early 
and accurate prediction of the disease. AD prediction deals with predictions of potential $\mathrm{AD}$ patients, in order to devise the strategic approaches towards treatment plans. Thus, it is very critical to develop such a model that accurately predicts the AD in its onset.

Disease prediction is referred as a binary classification problem. In binary classification, the answer to the problem is generated as one of the binary class. In the prediction of $\mathrm{AD}$, the patient data is taken as an input to the system and the system produces output as AD or Non-AD. In health domain, the instances of AD patients are very low as compared to the Healthy controls. This results in the different ratio of data for both $\mathrm{AD}$ as well as non- $\mathrm{AD}$, creating a problem of class imbalance. The classification results are always higher for the majority class hence produces biased results. Therefore, the class imbalance has to be handled by increasing the number of instances of AD class [9] [10] [11]. The objective is to develop a most suitable AD prediction model which helps to identify those patients who are most likely to develop this disease.

For achieving the aforementioned objective, A number of machine learning methods, such as Naïve Bayes [12] [13], Logit Boost [13], Support Vector Machines [12] [14]-[18], and Deep learning [15] [19] methods have been used in the previously proposed models to provide accurate prediction of AD patients [8]. However, these prior models cannot be used for prediction in the real world environment as they have a number of limitations.

Firstly, the feature selection methods adopted in majority of the previous models neglected the information rich variables present in different categories of the dataset for model development [20] [21]. Moreover, the prior work focused on diagnosis using imaging analysis [22] [23]. We argue that, early detection of AD using correlations among categorical data is far more challenging as compared to the diagnosis using imaging analysis. AD patient's data may not be complete and their data may also be large enough which makes it really hard for the prediction model to predict potential $\mathrm{AD}$ patients accurately.

Secondly, in previous work, the researchers have used only statistical methods for extracting the important features from the dataset [15] [24] [25]. Though these methods have been used successfully, application of these methods requires domain knowledge. Without the augmentation of the domain knowledge, these methods can yield erroneous results and such predictive model can show poor performance.

Thirdly, the researchers have used a very small set of data or a large data with so many missing values for prediction of the disease [5] [9] [10]. As the model gets trained too well over the small data hence results in model over-fitting. Moreover, with small data set, they lack the external testing and validation of their model and results. The large data with a huge number of missing values can produce unrealistic results while degrading the overall performance of the predictive model [26]. Therefore, a model is needed to be developed which can deal with big data set having missing values, class imbalance along with proper validation.

Finally, the evaluation measures used in the literature have mostly included the Accuracy of the classifier while neglecting the Recall and Area under the curve (AUC) [3]. The Recall showcases the ability of a predictive model to classify the 
percentage of $\mathrm{AD}$ patients in comparison to healthy controls. The AUC which is considered to be the baseline for evaluation of classifier's performance is rarely utilized for the prediction of $\mathrm{AD}$. A classification based model is considered good if it produces higher values for both of these measures [27]. Moreover, the integrated heterogeneous clinical and neuro-pathological data have been used by many researchers [13] [15] [19] [25]. However, the comparative study based on different classifiers applied to different categories of data for instance, clinical diagnosis (CD), clinical judgment of symptoms (CJS) and minimum mental state examination (MMSE) is still neglected. To the best of our knowledge, these three categories of AD data altogether have never been utilized in a single research for the early prediction of AD.

To overcome the aforementioned limitations, an AD prediction model has been proposed in this paper based on the different categories of data such as CD, CJS and MMSE. Initially in this model, the data is preprocessed for feature selection and class imbalance. The information rich variables are extracted using domain knowledge from all the three categories, while the data is then preprocessed for class imbalance. The percentage split validation is used to validate the data. Experiments are conducted to evaluate the performance of different classifiers in terms of Recall and AUC and compared with predominant classifiers used in literature. The experiments highlight the results of Naïve Bayes classifier model on CD category of data with higher TP rate (Recall) in comparison to other categories of data as well as models used in literature.

\section{Related Work}

Alzheimer's disease, most common in elderly people, need to be diagnosed on early stage. Nicolas Fayed et al (2012) highlighted the use of MRI scans (structural and functional) for increasing the prediction accuracy as compared to PET scans [28]. However it has been identified that structural MRI its self is insufficient for prediction of early AD and it needs to be combined with other biomarkers for better prediction accuracy and diagnosis.

Early diagnosis of AD occurs for less than half of the patients, while few are aware of the diagnosis. Kumar. B et al. examined the relationship of performance on cognitive tests for the diagnosis of AD on clinical basis [24]. They performed many tests based on episodic memory, executive function and global cognition over a sample of 2125 instances, out of which a percentage was African Americans and European Americans. This study highlighted that cognitive battery tests and specific cognitive function differences can diagnose AD up to 18years before the diagnosis in an actual population. The results also extended that one category of the dataset i.e. cognitive impairment can help in the diagnosis of AD in preclinical stage and can aid for early detection. While the midlife studies can provide better clinical diagnosis for early AD process.

Many potential drugs have failed to reduce the effect of AD progression in clinical diagnosis. K. Blennow et, al. introduced clinical utility in the prognosis of the disease [29]. The authors found that in the early stages only the temporal lobe is affected. The 
whole work was carried out using CSF modality. Authors defend the use of CSF for early detection. The clinical diagnosis method for the prognosis of AD show low classification accuracy having sensitivity ranging between $71 \%$ to $88 \%$ and specificities from $44 \%$ to $71 \%$ varied with the histo-pathologic criteria being used [30]. In the early clinical stages, the accuracy of the clinical diagnosis is very low. The use of MRI or PET scans combined with CSF biomarker based on results from information based questionnaires and memory tests will increase diagnostic accuracy of AD in its early stages [31] [32]. This study showed that the use of CSF biomarkers will enhance the specificity and sensitivity of AD signature that could lead to early prognosis of $\mathrm{AD}[33]$.

The accuracy of determining AD using clinical diagnosis with the help of clinical and neuropathological data is crucial. Thomas $\mathrm{G}$ Beach et al. used the dataset collected between 2005 and 2010 [34]. Initially the dataset had 1198 instances while it was reduced to 919 by eliminating the instances having missing values out of it. The dataset comprised of 271 normal patients (Nor) while remaining were probable/possible AD patients. After different observations and tests, researchers found that there were some cases which were classified as $\mathrm{AD}$ by clinical diagnosis but where classified as Non AD by histopathological threshold [35]. It was stated that clinical diagnosis of $A D$ varies depending upon the neuropathological and clinical method used while new biomarkers may increase the diagnostic accuracy of $\mathrm{AD}$ but will overlap between normal, demented and AD patients.

The pathologically proven data for the diagnosis of AD remained a concern. John M. Hoffman et al. presented a study on pathologic verification of $\mathrm{AD}$ with the role of specificity, sensitivity and diagnostic accuracy for detection of AD [36]. The authors used FDG PET brain scans of twenty two instances out of which 8 were women and 14 were men who had eventually pathologically confirmed for disease. As a result, clinical diagnosis was considered as the primary cause of disease detection in probable $\mathrm{AD}$ in 12 patients. The sensitivity and specificity of probable AD of clinical diagnosis were $63 \%$ and $100 \%$ while for possible AD were $75 \%$ and $100 \%$ respectively with the accuracy rate of $93 \%$ and $82 \%$. The authors concluded that metabolic patterns in FDG PET scans helps in differentiating dementia and can aid in clinical suspicion of $\mathrm{AD}$.

Computer aided diagnostic (CAD) tools can help the physicians for making better decision about the presence of abnormalities in human brain. In this paper, Veeramuthu et al. developed such tool for AD patients for diagnosis and they used PET dataset as experiment [37]. They defined such spatial normalization and intensity normalization as a pre-processing technique. Fisher Discriminant ratio (FDR) was used for feature extraction to develop Region of Interest (ROI). The author claimed the Recall of $82.67 \%$ with and overall accuracy of $91.33 \%$ in comparison to NFM+SV, VAF and MPCA+SVM.

Beside many classification techniques, Garam Lee et al used deep learning technique for the prediction of probable AD conversions [2]. The overall dataset of 753 instances was used, comprising of 338 AD patients while 415 were healthy controls. The authors combined demographics features along with the MRI data for prediction. The results revealed the overall accuracy of $81 \%$ with Recall of $84 \%$. However, they 
haven't done preprocessing of the data which is an important step especially in critical systems. Moreover, the authors have not utilized the other information rich variables of different categories of data along with demographics for the prediction of AD.

In comparison, Bhagya Shree et al used a CSID battery category of data set instead of most commonly used MMSE [25]. They have used a dataset having 51 features of $18 \mathrm{AD}$ patients and 448 Healthy Control. In their proposed model, they have used SMOTE to address the class imbalance followed by Naïve Bayes classifier for the evaluation of their model. This study shows over all accuracy of 96.6 percent with 96.7 Recall and 99.1 ROC, which are quite higher as compared to the previous studies. However, a comparative analysis with other set of classifiers and too small data set for experimentation can produce biased results.

\subsection{Critical evaluation measures}

In section 2, we provided the overview of literature based on the early diagnosis of AD using Machine learning techniques specially classification while the imaging modalities varied for instance, MRI, SPECT, and PET as shown in Table 1.

We critically analyzed each of these studies on the basis of certain important parameters such as:

Imaging modality used: This defined the type of imaging modality used by the researchers for AD detection. For instance PET scans, MRI scan or CSF etc.

No. of records used: This present the total number of samples selected for the experiment.

Classification technique used: We analyzed the methodology used for the prediction of AD. From these techniques, a comparative analysis is evaluated on the basis of Recall, ROC and computational time.

Table 1. Modalities that have been used to predict conversion from Healthy control to AD

\begin{tabular}{|l|l|}
\hline 1 & Memory \& Neuropsychological Tests (Manipulative WAIS subscales etc ) \\
\hline 2 & MR techniques (MR spectrometry, Tensor MRI etc) \\
\hline 3 & PET techniques (FDG PET, Amyloid PET etc) \\
\hline 4 & SPECT \\
\hline 5 & Laboratory TESTS (CSF, Plasma AB42, APOE genotype etc) \\
\hline 6 & Combination of above techniques (FDG-PET with APOE genotype etc) \\
\hline
\end{tabular}


Table 2. Data Set Details with critical evaluation

\begin{tabular}{|c|c|c|l|l|l|}
\hline Sr. \# & Ref & Data Set & \multicolumn{1}{|c|}{ Modality } & Feature Selection Approach & \multicolumn{1}{|c|}{ Classification Techniques } \\
\hline & {$[25]$} & 466 & No mentioned & Not Mentioned & Naïve Bayes \\
\hline & {$[27]$} & 131 & MRI & Voxel based & Hierarchical Ensemble \\
\hline & {$[2]$} & 753 & MRI & No & Deep learning \\
\hline & {$[21]$} & 86 & PET, MRI & AUC based FS & $\begin{array}{l}\text { DT, Naïve Bayes, SVM , TAN } \\
\text { Bayes }\end{array}$ \\
\hline & {$[16]$} & 383 & Not mentioned & No & LR \\
\hline & {$[26]$} & 776 & No & Random & $\begin{array}{l}\text { SVM, MLP, LR, RF, CART, } \\
\text { QDA, LDA }\end{array}$ \\
\hline$[13]$ & 260 & H-MRS & Random & $\begin{array}{l}\text { SVM, Random forest } \\
\text { BF Tree, FT, Logit boost, RF }\end{array}$ \\
\hline & {$[15]$} & 825 & MRI & VAF \& Regularized LR & SVM, LDS \\
\hline & {$[14]$} & 311 & MRI & Not Mentioned & $\begin{array}{l}\text { MK SVM, SK SVM, Deep } \\
\text { learning }\end{array}$ \\
\hline & {$[17]$} & 30 & EEG based & Not Mentioned & SVM \\
\hline $\begin{array}{c}\text { Proposed } \\
\text { Model }\end{array}$ & 101,696 & MRI & Domain Knowledge & $\begin{array}{l}\text { NB, J48, AdaBoostM1, } \\
\text { LogitBoost, Decision Stump, } \\
\text { SGD Text }\end{array}$ \\
\hline
\end{tabular}

\section{Proposed Model}

In this study, we proposed a model based on the findings of previous research carried out for the detection of $\mathrm{AD}$ in its early stages using classification techniques. In our research, instead of classification of whole dataset, few categories out of the dataset are obtained and are classified in the same way as the whole dataset. The detailed model of the classification algorithm implemented on the said categories to predict AD in its early stage is shown in Fig. 1.

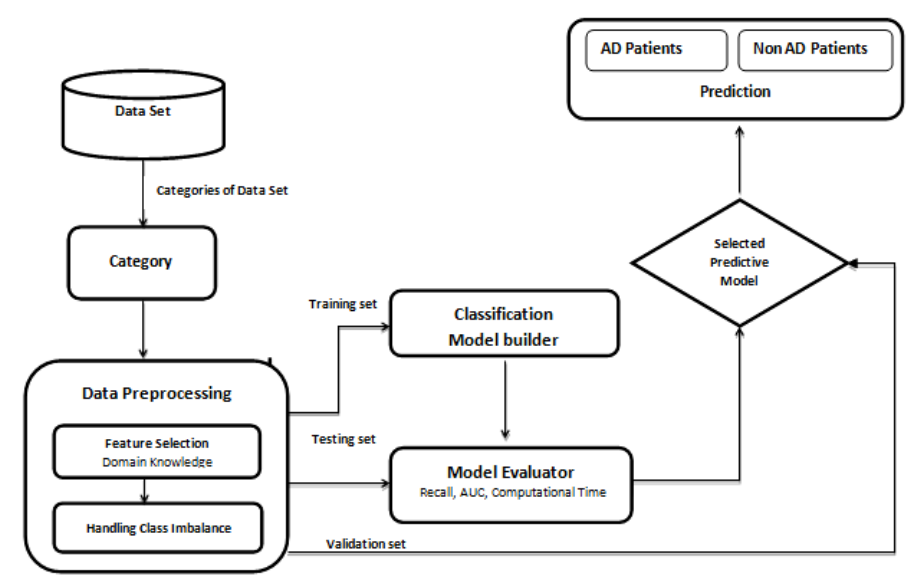

Fig. 1. Proposed model for the early detection of $\mathrm{AD}$ using Categorical data 


\subsection{Materials}

The data set used in this study comprised of 101696 instances of both men and women aged between 40 and 110. The Clinical Diagnosis and clinical judgment of symptoms and MMSE and Neuropsychological battery category initially had 78, 44 and 34 attributes respectively. These categories of data were classified as AD or Non $\mathrm{AD}$ on the basis of behavioral, physical and cognitive measures.

\subsection{Feature selection}

The categories contain a combination of features such as information rich variables and variables with least overall impact. The attributed with least impact can be removed from the dataset [23] [24] [29]. In this study, the variables having ratio of missing value more than $50 \%$ were removed from the targeted categories. Such kind of dimensional reduction can increase the overall prediction accuracy while decreasing the computational time. As a result, 30 information rich attributes of $\mathrm{CD}$ out of 78 attributes while 28 out of 44 variables of Clinical Judgment of symptoms category and 30 out of 34 attributes of MMSE category were obtained. The division of some common attributes of CD and CJS are shown in figure 1 and Figure 2. The detailed attributes of CD and CJS are explained in [38]. While the MMSE and Neuropsychological battery category have the following attributes MMSELOC, MMSELAN, MMSELANX, MMSEORDA, MMSEORLO, PENTAGON, MMSE, NPSYCLOC, NPSYLAN, NPSYLANX, LOGIMO, LOGIDAY, LOGIYR, LOGIPREV, LOGIMEM, DIGIF, DIGIFLEN, DIGIB, DIGIBLEN, ANIMALS, VEG, TRAILA, TRAILARR, TRAILALI, TRAILB, TRAILBRR, TRAILBLI, WAIS, MEMUNITS, MEMTIME, BOSTON and COGSTAT. This category had least missing values which resulted in equal contribution for the disease prediction.

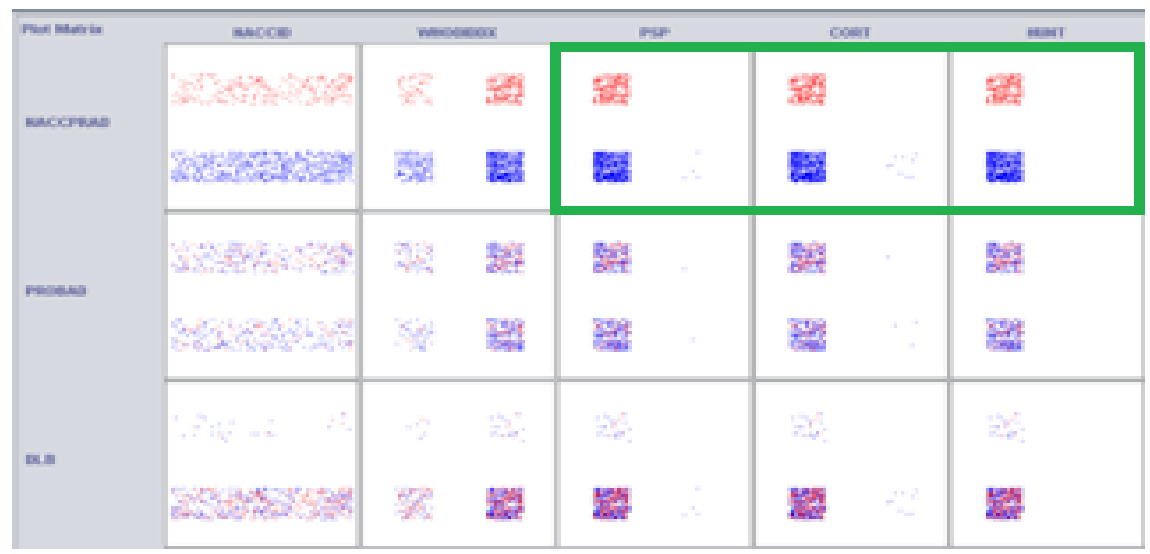

Fig. 2. Visualization of some prominent features in the CD dataset. 


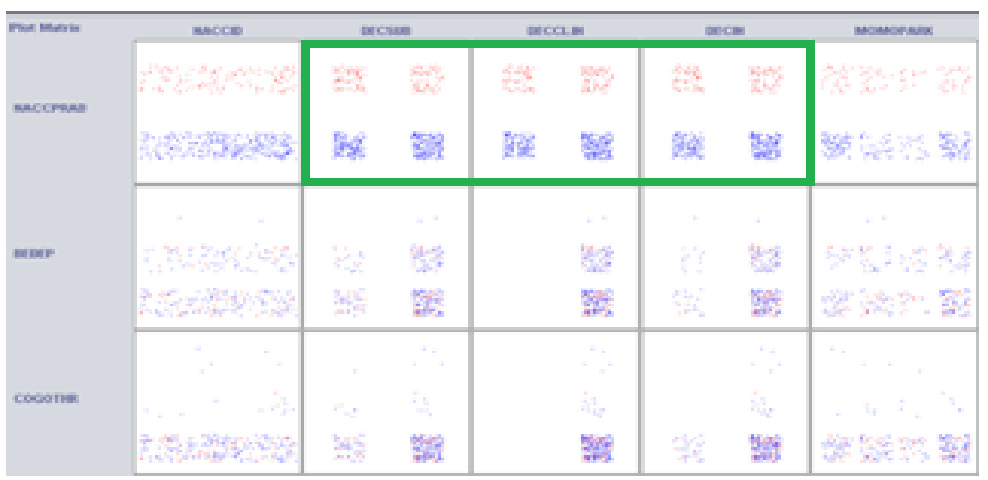

Fig. 3. Visualization of some prominent features in the CJS dataset.

\subsection{Class imbalance}

Class imbalance issue can occur in binary classification when one class is in majority while other class is having less number of instances. With such imbalance, the classifiers tend to misclassify the minority class while producing good results for the majority class [12]. There are many class imbalance algorithms such as SMOTE and Class Balancer algorithms. However, for this study, the class imbalance issue is dealt by using class balancing algorithm on the selected categorical data, which works by assigning equal weights to both classes while the instance ratio is maintained.

\subsection{Classification \& evaluation}

Different well known classifiers are then applied to classify the reduced categorical data, obtained in the previous step. Although, many different classification methods exists while Naïve Bayes and SVM being the most popular, for this study we have done a comparative analysis of most common classifiers such as Naïve Bayes, J48, Decision Stump, LogitBoost and AdaBoost. We used a 70-30 percentage split for our study and validated the results with unseen data. Moreover, the evaluation measure for this study were Recall (Sensitivity), Training time of algorithm, Area under the curve (AUC) of an algorithm as depicted in Table 3.

Table 3. Comparison of classification performance using Category I: CD in identification of two groups of Norm and AD

\begin{tabular}{|l|c|c|c|}
\hline \multicolumn{1}{|c|}{ Classifier } & Recall & ROC & Computational Time \\
\hline Naïve Bayes & 0.967 & 0.99 & 0.08 \\
\hline Decision Stump & 0.929 & 0.927 & 0.4 \\
\hline AdaBoostM1 $1_{\text {S S }}$ & 0.94 & 0.958 & 3.76 \\
\hline LogitBoost (Meta) & 0.955 & 0.971 & 2.41 \\
\hline SGD Text & 0.76 & 0.5 & 12.06 \\
\hline J48 & 0.961 & 0.982 & 6.59 \\
\hline
\end{tabular}


Table 4. Comparison of classification performance using Category II: MMSE in identification of two groups of Norm and $\mathrm{AD}$

\begin{tabular}{|l|c|c|c|}
\hline \multicolumn{1}{|c|}{ Classifier } & Recall & ROC & Computational Time \\
\hline Naïve Bayes & 0.845 & 0.916 & 0.06 \\
\hline Decision Stump & 0.805 & 0.798 & 0.03 \\
\hline AdaBoostM1 D & 0.83 & 0.905 & 1.02 \\
\hline LogitBoost (Meta) & 0.955 & 0.971 & 2.41 \\
\hline SGD Text & 0.5 & 0.5 & 3.7 \\
\hline J48 & 0.85 & 0.892 & 1.5 \\
\hline
\end{tabular}

Table 5. Comparison of classification performance using Category III: CJS in identification of two groups of Norm and AD

\begin{tabular}{|l|c|c|c|}
\hline \multicolumn{1}{|c|}{ Classifier } & Recall & ROC & Computational Time \\
\hline Naïve Bayes & 0.866 & 0.941 & 0.07 \\
\hline Decision Stump & 0.522 & 0.520 & 0.1 \\
\hline AdaBoostM1 D & 0.522 & 0.522 & 1.54 \\
\hline LogitBoost (Meta) & 0.522 & 0.523 & 1.37 \\
\hline SGD Text & 0.5 & 0.5 & 10.03 \\
\hline J48 & 0.878 & 0.934 & 4.35 \\
\hline
\end{tabular}

\section{Discussion}

In the current paper, we have proposed a classification based model using different categories of data including feature selection, class balancing, and validation. We have reviewed recent studies in the field of $\mathrm{AD}$ prediction that have used machine learning for classification as shown in Table 2. The review of literature and experimental results indicates that prediction of disease such as AD using Naïve Bayes classifier had the best prediction performance based on the selected categorical attributes. The point to be observed is that it is difficult to compare the performances of different classifiers due to the different set of features, different data sets with varying sizes; the performance of the Recall tends to be around $70-95 \%$. However, the fully validated large datasets resulted in mostly lower accuracies. The small data sets usually results in higher prediction accuracies and have an over-fitted model. Other way round, they may have used the clinical measures of diagnosis as an input to their system which lead to circularity problems. Moreover, the different multivariate classifier over same input size from same cohort tends to perform similarly. Finally, the results shows that NB can perform better than any other methods that are reported in Table 2 $\&$ 6. Moreover, its use can be very helpful for the physicians in disease the prediction. Our findings highlight the potential of NB for disease prediction over other methods. 
Table 6. Overall comparison of results based on the Best classifier in the literature and proposed approach.

\begin{tabular}{|c|l|l|l|l|c|c|c|}
\hline Ref. \# & Data Set Sample & \multicolumn{1}{|c|}{$\begin{array}{c}\text { Class } \\
\text { Imbalance }\end{array}$} & Validation & $\begin{array}{c}\text { Best } \\
\text { Classifier }\end{array}$ & Recall & AUC & $\begin{array}{c}\text { Training } \\
\text { Time }\end{array}$ \\
\hline$[25]$ & $\begin{array}{l}448 \mathrm{Nor} \\
18 \mathrm{AD}\end{array}$ & SMOTE & No & Naïve Bayes & 96.7 & 96.6 & No \\
\hline$[27]$ & $\begin{array}{l}70 \mathrm{AD} \\
61 \mathrm{Nor}\end{array}$ & No & 10 fold CV & Ensemble & 86.5 & No & No \\
\hline$[2]$ & $\begin{array}{l}338 \mathrm{AD} \\
415 \mathrm{Nor}\end{array}$ & No & No & $\begin{array}{l}\text { Deep learn- } \\
\text { ing }\end{array}$ & 0.84 & No & No \\
\hline$[12]$ & 677 & SMOTE & HO CV & Naïve Bayes & No & No & No \\
\hline$[21]$ & 41 AD, 45 Nor & No & 10 fold CV & LR & No & 88.7 & No \\
\hline$[16]$ & 383 Nor 121 AD & No & 5 Fold CV & SVM & 64 & 73 & No \\
\hline$[26]$ & 229 Nor 397 AD & No & No & RF & 89.1 & 94.3 & No \\
\hline$[13]$ & 99 Nor 161 AD & No & 10 Fold CV & SLP & 86.1 & 86.6 & No \\
\hline$[15]$ & 200 AD, 231 Nor & No & 10 fold CV & SVM & 87 & 90 & No \\
\hline$[14]$ & 77 Nor, 234 AD & No & 10 Fold CV & Multi Layer & 88.57 & No & No \\
\hline$[17]$ & 15 Nor, 15 AD & No & No & SVM & Yes & No & No \\
\hline $\begin{array}{l}\text { Proposed } \\
\text { Model }\end{array}$ & 7595 Nor, & $\begin{array}{l}\text { Class } \\
\text { Balancer }\end{array}$ & $\begin{array}{l}\text { Percentage } \\
\text { Split }\end{array}$ & NB & 96.4 & 99.2 & 0.08 \\
\hline
\end{tabular}

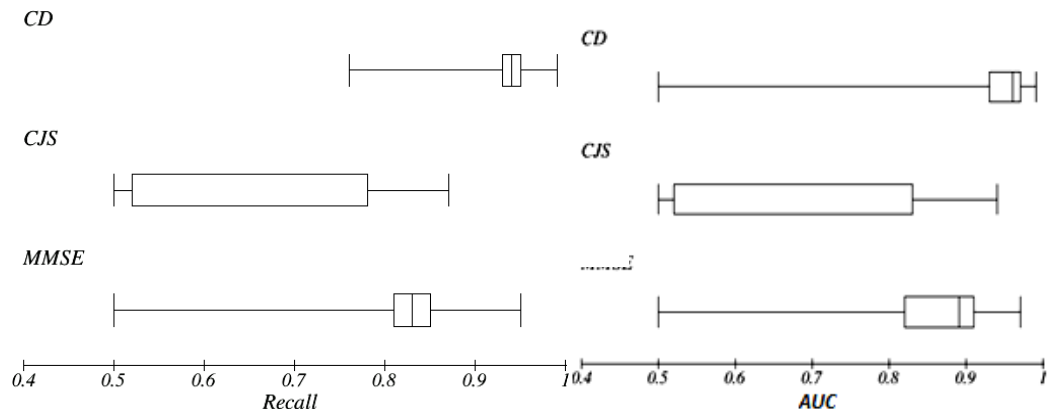

Fig. 4. Box Plot for the comparisons of three categories on the basis of:
(a) Recall
(b) AUC

\section{Conclusion}

The research is based on the prediction and prognosis of $\mathrm{AD}$ using classification algorithms over different categories of data. The information rich categories of the dataset were preprocessed and passed through different classifiers for evaluation. The results shown in fig 4(a) \& (b) demonstrate that Clinical Diagnosis category provides better prediction for the most commonly used classifiers. Moreover Naïve Bayes outperformed the results of other classifiers as well as the results obtained in literature with higher Recall and AUC. To conclude, different classifier more specifically Naïve 
Bayes applied over categorical data have a great potential for being implemented in clinical practice to aid $\mathrm{AD}$ diagnosis.

\section{Acknowledgement}

The NACC database is funded by NIA/NIH Grant U01 AG016976. NACC data are contributed by the NIAfunded ADCs: P30 AG019610 (PI Eric Reiman, MD), P30 AG013846 (PI Neil Kowall, MD), P50 AG008702 (PI Scott Small, MD), P50 AG025688 (PI Allan Levey, MD, PhD), P30 AG010133 (PI Andrew Saykin, PsyD), P50 AG005146 (PI Marilyn Albert, PhD), P50 AG005134 (PI Bradley Hyman, MD, PhD), P50 AG016574 (PI Ronald Petersen, MD, PhD), P50 AG005138 (PI Mary Sano, PhD), P30 AG008051 (PI Steven Ferris, PhD), P30 AG013854 (PI M. MarselMesulam, MD), P30 AG008017 (PI Jeffrey Kaye, MD), P30 AG010161 (PI David Bennett, MD), P30 AG010129 (PI Charles DeCarli, MD), P50 AG016573 (PI Frank LaFerla, PhD), P50 AG016570 (PI David Teplow, PhD), P50 AG005131 (PI Douglas Galasko, MD), P50 AG023501 (PI Bruce Miller, MD), P30 AG035982 (PI Russell Swerdlow, MD), P30 AG028383 (PI Linda Van Eldik, PhD), P30 AG010124 (PI John Trojanowski, MD, PhD), P50 AG005133 (PI Oscar Lopez, MD), P50 AG005142 (PI Helena Chui, MD), P30 AG012300 (PI Roger Rosenberg, MD), P50 AG005136 (PI Thomas Montine, MD, PhD), P50 AG033514 (PI Sanjay Asthana, MD, FRCP), and P50 AG005681 (PI John Morris, MD).

\section{$7 \quad$ References}

[1] Stamps, J. J., Bartoshuk, L. M., \& Heilman, K. M. (2013). A brief olfactory test for Alzheimer's disease. Journal of the neurological sciences, 333(1-2), 19-24. LR8. https://doi.org/10.1016/j.jns.2013.06.033

[2] Lee, G., Nho, K., Kang, B., Sohn, K. A., \& Kim, D. (2019). Predicting Alzheimer's disease progression using multi-modal deep learning approach. Scientific reports, 9(1), 1952. LR6. https://doi.org/10.1038/s41598-018-37769-Z

[3] Zhang, R., Simon, G., \& Yu, F. (2017). Advancing Alzheimer's research: A review of big data promises. International journal of medical informatics, 106, 48-56. LR15. https://doi.org/10.1016/j.ijmedinf.2017.07.002

[4] Bookheimer SY, Strojwas MH, Cohen MS, Saunders AM, Pericak-Vance MA, Mazziotta $\mathrm{JC}$, et al. Patterns of brain activation in people at risk of Alzheimer's disease. N Engl J Med 2000;343:450-6. https://doi.org/10.1056/NEJM200008173430701

[5] Petrella JP, Wang L, Krishnan S, Slavin MJ, Prince SE, Tran TT, et al. Cortical deactivation in mild cognitive impairment: high-field strength functional MR imaging. Radiology 2007;245:224-35. https://doi.org/10.1148/radiol.2451061847

[6] Supekar K, Menon V, Rubin D, Musen M, Greicius MD. Network analysis of intrinsic functional brain connectivity in Alzheimer's disease. PLoSComputBiol 2008;4(6):1-11. https://doi.org/10.1371/journal.pcbi.1000100

[7] Farhan, S., M. A. Fahiem, et al. (2014). "An Ensemble-of-Classifiers Based Approach for Early Diagnosis of Alzheimer's Disease: Classification Using Structural Features of Brain 
Images." Computational and mathematical methods in medicine.https://doi.org/10.1155/ 2014/862307

[8] Dallora, A. L., Eivazzadeh, S., Mendes, E., Berglund, J., \& Anderberg, P. (2017). Machine learning and microsimulation techniques on the prognosis of dementia: A systematic literature review. PloS one, 12(6), e0179804.LR9 https://doi.org/10.1371/journal.pone.0179804

[9] SY Bookheimer, MH Strojwas, MS Cohen, AM Saunders, MA Pericak-Vance, JC Mazziotta et al., "Patterns of brain activation in people at risk of Alzheimer's disease", N Engl J Med, vol. 6, pp. 343-450, 2000. https://doi.org/10.1371/journal.pone.0179804

[10] K Supekar, V Menon, D Rubin, M Musen, MD Greicius, "Network analysis of intrinsic functional brain connectivity in Alzheimer's disease", PLoS Comput Biol 4, no. 6, pp. 211, 2008. https://doi.org/10.1371/journal.pcbi.1000100

[11] J.A. Cruz, D.S. Wishart, "Applications of Machine Learning in Cancer Prediction and Prognosis", Cancer Informatics, vol. 2, pp. 59-77, 2006. https://doi.org/10.1177/11769351 0600200030

[12] Nunes, C., Silva, D., Guerreiro, M., de Mendonça, A., Carvalho, A. M., \& Madeira, S. C. (2013, September). Class Imbalance in the Prediction of Dementia from Neuropsychological Data. In Portuguese Conference on Artificial Intelligence (pp. 138-151). Springer Berlin Heidelberg. https://doi.org/10.1007/978-3-642-40669-0_13

[13] Munteanu, C. R., Fernandez-Lozano, C., Abad, V. M., Fernández, S. P., Álvarez-Linera, J., Hernández-Tamames, J. A., \& Pazos, A. (2015). Classification of mild cognitive impairment and Alzheimer's Disease with machine-learning techniques using $1 \mathrm{H}$ Magnetic Resonance Spectroscopy data. Expert Systems with Applications, 42(15), 6205-6214. https://doi.org/10.1016/j.eswa.2015.03.011

[14] Liu, S., Liu, S., Cai, W., Pujol, S., Kikinis, R., \& Feng, D. (2014, April). Early diagnosis of Alzheimer's disease with deep learning. In Biomedical Imaging (ISBI), 2014 IEEE 11th International Symposium on (pp. 1015-1018). IEEE. https://doi.org/10.1109/ISBI.2014. $\underline{6868045}$

[15] Moradi, E., Pepe, A., Gaser, C., Huttunen, H., Tohka, J., \& Alzheimer's Disease Neuroimaging Initiative. (2015). Machine learning framework for early MRI-based Alzheimer's conversion prediction in MCI subjects. Neuroimage, 104, 398-412. https://doi.org/10.1016/ j.neuroimage.2014.10.002

[16] Maroco, J., Silva, D., Rodrigues, A., Guerreiro, M., Santana, I., \& de Mendonça, A. (2011). Data mining methods in the prediction of Dementia: A real-data comparison of the accuracy, sensitivity and specificity of linear discriminant analysis, logistic regression, neural networks, support vector machines, classification trees and random forests. BMC research notes, 4(1), 299. https://doi.org/10.1186/1756-0500-4-299

[17] Zhao, Y., \& He, L. (2014, November). Deep learning in the EEG diagnosis of Alzheimer's disease. In Asian Conference on Computer Vision (pp. 340-353). Springer International Publishing. https://doi.org/10.1007/978-3-319-16628-5_25

[18] Westman, E., J.-S. Muehlboeck, et al. (2012). "Combining MRI and CSF measures for classification of Alzheimer's disease and prediction of mild cognitive impairment conversion." Neuroimage 62(1): 229-238. https://doi.org/10.1016/j.neuroimage.2012.04.056

[19] Jo, T., Nho, K., \& Saykin, A. J. (2019). Deep Learning in Alzheimer's disease: Diagnostic Classification and Prognostic Prediction using Neuroimaging Data. arXiv preprint arXiv:1905.00931.LR7.https://doi.org/10.3389/fnagi.2019.00220

[20] Wordoffa, H., \& Wangoria, E. (2012). Alzheimer's Disease Stage Prediction using Machine Learning and Multi Agent System.

[21] Escudero, J., Ifeachor, E., Zajicek, J. P., Green, C., Shearer, J., Pearson, S., \& Alzheimer's Disease Neuroimaging Initiative. (2013). Machine learning-based method for personalized 
and cost-effective detection of Alzheimer's disease. IEEE transactions on biomedical engineering, 60(1), 164-168. https://doi.org/10.1109/TBME.2012.2212278

[22] EF LA. Petricoin Liotta, "serum proteomic pattern diagnostics for early detection of cancer", Curr Opin Biotechnol, vol. 15, pp. 24-30, 2004. https://doi.org/10.1016/j.copbio. $\underline{2004.01 .005}$

[23] L Bocchi, G Coppini, J Nori, G Valli, "Detection of single and clustered microcalcifications in mammograms using fractals models and neural networks", Med Eng Phys, vol. 26, pp. 303-12, 2004. https://doi.org/10.1016/j.medengphy.2003.11.009

[24] Rajan, K. B., Wilson, R. S., Weuve, J., Barnes, L. L., \& Evans, D. A. (2015). Cognitive impairment 18 years before clinical diagnosis of Alzheimer disease dementia. Neurology, 85(10), 898-904. https://doi.org/10.1212/WNL.0000000000001774

[25] Shree, S. B., \& Sheshadri, H. S. (2018). Diagnosis of Alzheimer's disease using naive Bayesian classifier. Neural Computing and Applications, 29(1), 123-132.LR11. https://doi.org/10.1007/s00521-016-2416-3

[26] Campos, S., Pizarro, L., Valle, C., Gray, K.R., Rueckert, D., \& Allende, H. (2015). Evaluating Imputation Techniques for Missing Data in ADNI: A Patient Classification Study.CIARP. https://doi.org/10.1007/978-3-319-25751-8_1

[27] Huang, M., Yang, W., Feng, Q., Chen, W., Weiner, M. W., Aisen, P., \& Toga, A. W. (2017). Longitudinal measurement and hierarchical classification framework for the prediction of Alzheimer's disease. Scientific reports, 7, 39880.LR13. https://doi.org/10. $1038 /$ srep39880

[28] Fayed, N., Modrego, P. J., Salinas, G. R., \& Gazulla, J. (2012). Magnetic resonance imaging based clinical research in Alzheimer's disease. Journal of Alzheimer's Disease, 31(s3), S5-S18. https://doi.org/10.3233/JAD-2011-111292

[29] Blennow, K., Dubois, B., Fagan, A. M., Lewczuk, P., de Leon, M. J., \& Hampel, H. (2015). Clinical utility of cerebrospinal fluid biomarkers in the diagnosis of early Alzheimer's disease. Alzheimer's \& Dementia, 11(1), 58-69. https://doi.org/10.101 6/j.jalz.2014.02.004

[30] Beach, T. G., Monsell, S. E., Phillips, L. E., \& Kukull, W. (2012). Accuracy of the clinical diagnosis of Alzheimer disease at National Institute on Aging Alzheimer Disease Centers, 2005-2010. Journal of neuropathology \& experimental neurology, 71(4), 266-273. https://doi.org/10.1097/NEN.0b013e31824b211b

[31] Teipel, S. J., Sabri, O., Grothe, M., Barthel, H., Prvulovic, D., Buerger, K., \& Hampel, H. (2013). Perspectives for multimodal neurochemical and imaging biomarkers in Alzheimer's disease. Journal of Alzheimer's Disease, 33(s1). https://doi.org/10.3233/JAD-2012$\underline{129030}$

[32] Galvin, J. E., Fagan, A. M., Holtzman, D. M., Mintun, M. A., \& Morris, J. C. (2010). Relationship of dementia screening tests with biomarkers of Alzheimer's disease. Brain, 133(11), 3290-3300. https://doi.org/10.1093/brain/awq204

[33] Fagan, A. M., Roe, C. M., Xiong, C., Mintun, M. A., Morris, J. C., \& Holtzman, D. M. (2007). Cerebrospinal fluid tau/?-amyloid42 ratio as a prediction of cognitive decline in nondemented older adults. Archives of neurology, 64(3), 343-349. https://doi.org/10.1 001/archneur.64.3.noc60123

[34] Beach, T. G., Monsell, S. E., Phillips, L. E., \& Kukull, W. (2012). Accuracy of the clinical diagnosis of Alzheimer disease at National Institute on Aging Alzheimer Disease Centers, 2005-2010. Journal of neuropathology \& experimental neurology, 71(4), 266-273. https://doi.org/10.1097/NEN.0b013e31824b211b

[35] Nelson, P. T., Kukull, W. A., \& Frosch, M. P. (2010). Thinking outside the box: Alzheimer-type neuropathology that does not map directly onto current consensus recommen- 
dations. Journal of Neuropathology \& Experimental Neurology, 69(5), 449-454. https://doi.org/10.1097/NEN.0b013e3181d8db07

[36] Hoffman, J. M., Welsh-Bohmer, K. A., Hanson, M., Crain, B., Hulette, C., Earl, N., \& Coleman, R. E. (2000). FDG PET imaging in patients with pathologically verified dementia. Journal of Nuclear Medicine, 41(11), 1920-1928.

[37] Veeramuthu, A., S. Meenakshi, et al. (2014). A New Approach for Alzheimer's disease Diagnosis by using Association Rule over PET Images. International Journal of Computer Applications 91(9), 9-14. https://doi.org/10.5120/15908-5009

[38] Khan, A., \& Usman, M. (2018, April). Early Diagnosis of Alzheimer's Disease Using Informative Features of Clinical Data. In Proceedings of the International Conference on Machine Vision and Applications (pp. 56-60). ACM. https://doi.org/10.1145/3220511. $\underline{3220515}$

\section{Authors}

Aunsia Khan is a PhD student and a Lecturer at Department of Computing at Shaheed Zulfikar Ali Bhutto Institute of Science and Technology, Islamabad, Pakistan. Her research area includes data mining and Machine learning for disease prediction. She is working on Alzheimer's disease prediction from past 4 years. Email: aunsia@szabist-isb.edu.pk

Dr. Muhammad Usman is currently an Associate Professor of Computer Science in the Department of Computing at Shaheed Zulfikar Ali Bhutto Institute of Science and Technology, Islamabad, Pakistan. His research interests include Data Mining, Data Warehousing, OLAP, Business Intelligence and Knowledge discovery. He has served as reviewer for a number of premier journals and conferences, including the International Journal of Knowledge-based Systems and International Journal of Expert Systems with Applications.

Article submitted 2019-08-08. Resubmitted 2019-09-23. Final acceptance 2019-09-23. Final version published as submitted by the authors. 